\title{
Letramento digital de pessoas com necessidades específicas
}

\author{
Ciro Ferreira de Carvalho Júnior \\ Universidade de Salvador (UNIFACS) \\ (cirofcjr@ifto.edu.br)
}

\author{
Kely Rejane Souza dos Anjos de Carvalho \\ Universidade Federal do Tocantins (UFT) \\ (kelyrejanecarvalho@gmail.com)
}

Resumo: Este artigo tem como escopo principal compartilhar reflexões a partir da compreensão da literatura sistematizada sobre as tecnologias na atual conjuntura social, e o quanto tal conhecimento pode ser capaz de impactar no processo ensino-aprendizagem de pessoas com necessidades específicas. Desta forma, apresentamos inicialmente uma metodologia bibliográfica a partir da contribuição de diferentes autores ligados ao uso da tecnologia, no sentido de promover um diálogo mais interdisciplinar. Como aporte teórico, procuramos mobilizar saberes de diferentes percepções do conhecimento humano, aplicados a temática deste artigo, com o intuito de verificarmos o mesmo objeto sob vários pontos de vista. Refletiremos a tecnologia como ferramenta necessária e indispensável para melhorar a inter-relação entre as pessoas, possibilitando maior inclusão e qualidade de vida. Desta forma, as ferramentas metodológicas são consideradas um mecanismo essencial para que esta inclusão, de fato ocorra.

Palavras-chave: Tecnologia; Aprendizagem; Inclusão; Letramento Digital; Necessidades Específicas.

Digital literacy of people with specific needs

Abstract: This article's main goal is to share reflections on the literature of the systematization of the technologies in the social current conjuncture and how such knowledge can be able to impact the teaching-learning processes of people with specific needs. Thus, firstly we present a bibliographical methodology with the contribution of different authors related to the use of technology in order to promote a more interdisciplinary dialogue. Using a theoretical contribution, we seek to mobilize expertise of different perceptions of human knowledge applied to the this article's theme, in order to verify the same object from various points of view. We will reflect technology as a necessity and indispensable machine to improve the interrelationship between people, enabling greater inclusion and quality of life. Along these lines the methodological tools are an essential mechanism for this inclusion to in fact occurs.

Keywords: Technology; Learning; Inclusion; Digital Literacy; Specific Needs.

\section{INTRODUÇÃO}


No presente artigo apresentaremos um panorama das tecnologias da era pósmoderna e o quanto tais tecnologias podem impactar no processo de ensinoaprendizagem de pessoas com necessidades específicas. A pós modernidade é um período gerador de diversas reflexões e investigações, tais como: a tecnologia como ferramenta basilar para a educação, a inclusão e exclusão social de indivíduos que são engolidos pela era informatizada, pelos impactos sociais, econômicos e, mesmo, ambientais que as tecnologias podem causar em detrimento de um contexto mais harmônico entre as partes.

Neste artigo discutiremos sobre inclusão e exclusão sociais e digitais, principalmente de pessoas com necessidades específicas. Acreditamos que o conhecimento e acesso a ferramentas tecnológicas, que permitam a inclusão digital, trazem diversos benefícios no dia a dia, desde o convívio social até mesmo o acesso das pessoas com necessidades específicas ao mercado de trabalho.

A perspectiva de reflexão que abordamos sobre educação neste trabalho, não se refere ao sentido de educação formal, como preconizado na Lei de Diretrizes e Bases da Educação (LDB), mas sim, no sentido dos usuários saberem lidar com a referida ferramenta que, com o advento da globalização, tem ganhado mais espaço e, com isso, tem exercido papel importante em diferentes classes sociais, podendo servir como instrumento de inclusão social de pessoas com necessidades específicas, sejam elas físicas ou intelectuais, como autismo, por exemplo. Em outras palavras, nos referimos ao fato de haver, ou não, exclusão por parte das pessoas que desconhecem o manuseio das ferramentas básicas do contexto informacional e/ou computacional, recursos estes, que podem auxiliar em várias áreas do conhecimento, como também na área da educação, que no nosso caso é voltado para educação inclusiva de pessoas com necessidades específicas.

A metodologia desenvolvida neste artigo é do tipo bibliográfica, tendo em vista que é elaborada uma revisão de literatura especializada na área, colocando-as em confluência. Para tanto, foram utilizados, como principais autores, Oliveira et al (S/D), Souza (2008), Zanata e Carvalho (2008), Teixeira e Marcon (2009), Turci (2012), Aires et al (2014), e Moreira et al (2015).

A discussão entre diferentes autores contribui na construção de uma percepção mais global sobre o assunto proposto, incentivando o diálogo e a construção de ideias 
autônomas a partir de tais confrontos teóricos. Por isso, trata-se de um artigo de relevância teórica bastante pertinente.

Além desta Introdução, das Considerações Finais e das Referências, este artigo é constituído pelas seções Tecnologias na Aprendizagem, Letramento Digital, Tecnologia, Exclusão e Inclusão Digital em Contextos de Crianças com Autismo e Trabalhos Futuros.

\section{TECNOLOGIAS NA APRENDIZAGEM}

Aprender, na contemporaneidade, é um processo diretamente associado às novas concepções de mundo e linguagem, partindo do princípio de que a aprendizagem não se esgota mais na esfera escolar. A ideia de aprendizagem está diluída a qualquer contexto que se faça pertinente o compartilhamento de conhecimento. É nesse sentido, que a tecnologia aparece como grande revolucionária nesse campo, tendo em vista a importância que se é dada às novas mídias sociais da atualidade, tais como redes sociais, YouTube, etc.

Para Turci e Costa (2012), é necessário entender que a aprendizagem sobre tecnologia se faz cada vez mais importante e, para isso, é necessário que seja ampliado o leque de discussões sobre uso de softwares e outros elementos da informática, considerando que o computador é ferramenta fundamental para a interação desses novos tempos.

Dentro desse âmbito, é necessário entender a tecnologia como algo motivacional e não como uma ferramenta excludente. Seguindo a primeira concepção, entende-se que a tecnologia desperta a curiosidade do sujeito e, com isso, incentivao a ir além do que lhe é proposto. Isso, por sua vez, ajuda na formação de alguém mais autônomo e, com isso, mais sagaz na prática dos estudos (ZANATA, CARVALHO, 2008).

Assim, a aprendizagem, por meio de ferramentas tecnológicas, torna-se o pivô da democratização do saber, sobretudo no que se refere à autonomia das instituições e a rapidez com a qual as informações são compartilhadas. Nesse sentido, funciona como uma culminância de fatores facilitadores. 
Entretanto, há de se frisar que este teor facilitador só é possível desde que essa tecnologia seja inclusiva, no sentido de que proporcione o acesso de pessoas de todos os tipos, de todas as escolaridades e, principalmente, com qualquer tipo de deficiência (TEIXEIRA, MARCON, 2009).

Acreditar em uma aprendizagem tecnologicamente inclusiva ainda é um fator bastante preocupante no Brasil, tendo em vista a realidade social, cultural e econômica que ainda é bastante divergente no país. No entanto, casos isoladospodem ser estudados conforme cada região. Questões sobre inclusão e exclusão por meio da tecnologia serão mais explanadas nas próximas seções.

\section{LETRAMENTO DIGITAL}

Compreender os meios tecnológicos como um aporte capaz de incluir um indivíduo em um contexto social maior é reconhecer, consequentemente, que os meios sociais são permeados por situações interacionais que demandam conhecimento nessa área. Diante dessa realidade, há de se considerar o que se tem chamado de letramento digital, ou letramento tecnológico, muito se faz necessário em todos os contextos de interação, sobretudo naqueles que se munem da tecnologia e informática para se propagarem com mais exatidão.

Entende-se o letramento digital como um conjunto de habilidades reflexivas sobre o papel da informática e de seus recursos a partir da ideia de interação e caracterização social (SILVA, 2014). Nesse sentido, as diversas ferramentas tecnológicas não podem ser pensadas fora de uma situação de uso, tendo em vista que os homens se comunicam, das mais diversas formas, por meio delas.

Conversas em redes sociais, por exemplo, é o que há de mais básico sobre isso. $\mathrm{Na}$ atualidade, procedimentos, muitas vezes complexos, podem ser resolvidos a partir de aplicativos que viabilizam o andamento de processos costumeiramente mais demorados. Nesse sentido, fala-se que a prática de letramento digital, ou tecnológico, é, sem dúvida, uma maneira de tornar mais estreita a barreira que distancia os sujeitos sociais.

Associar a educação e a computação de forma a poder incluir pessoas com necessidades específicas no convívio com as tecnologias atuais, pode ser 
considerado como educação inclusiva. Neste contexto, ao incentivar crianças e adolescentes com necessidades específicas, tanto físicas como intelectuais, a ter um contato, por mais básico que seja, com as ferramentas tecnológicas, poderíamos ter um melhor resultado no processo ensino-aprendizagem com inclusão digital.

A necessidade de se ter uma prática letrada no universo tecnológico, inclusive, pode se tornar um agente catalisador da inclusão social e digital, tendo em vista que o conhecimento crítico-reflexivo da tecnologia, enquanto linguagem, é base para o andamento e compreensão social.

Para que as tecnologias digitais mais inovadoras se tornem, efetivamente, mediadoras do processo de inclusão do ensino-aprendizagem é necessário que a prática de letramento seja algo capaz de permear essas relações, estabelecendo sentidos, coerências e significados construídos pelo próprio usuário da tecnologia. Isso, por sua vez, deve despertar e instruir seus passos para lidar com situações vindouras, mas que solicitam o mesmo capricho com a ferramenta ora mencionada.

\section{TECNOLOGIA, EXCLUSÃO E INCLUSÃO DIGITAL EM CONTEXTOS DE CRIANÇAS COM AUTISMO}

Para Aires et al (2014), "É importante saber como os indivíduos autistas se comunicam com os outros, qual a linguagem desenvolvida por eles e como é a sua forma de aprendizagem". Mesmo com bastantes pesquisas na área, não se pode definir, ainda, claramente o autismo. Há estudos que definem o autismo como sendo um transtorno orgânico e não genético.

Entende-se por autismo um conjunto de transtornos intelectuais, capazes de alterar o funcionamento cerebral, o que acarreta numa disjunção na interação social e a comunicação de natureza verbal e não-verbal. Diante disso, afirma-se que "o foco da proteção aos direitos dos autistas não está mais na criação de novos instrumentos normativos, eis que a aplicação do que está previsto na Convenção seria suficiente para tornar mais agradável e digna à sua vida e de seus familiares" (MODALOZZO, 2011, p. 6).

Uma grande dificuldade que pode ser encontrada no momento do processo de ensino-aprendizagem de uma pessoa diagnosticada com autismo é que ela vive em 
seu "próprio mundo virtual". Contudo é de extrema importância a convivência com pessoas do "mundo real". Seria nesse estreitamento de laços que ferramentas tecnológicas poderiam contribuir.

Muitas pesquisas têm problematizado a questão da tecnologia como maneira de incluir crianças com autismo, ou outras deficiências, sobretudo físicas. Dentre os trabalhos, podem-se citar as pesquisas de Oliveira et al (S/D), Souza (2008), Zanata e Carvalho (2008), Teixeira e Marcon (2009), Turci (2012), Aires et al (2014), e Moreira et al (2015).

Todas as pesquisas listadas problematizam a dificuldade de pessoas cegas, surdas, com ausência de fala, ou com qualquer outra deficiência física, a terem acesso a um ensino digno e de qualidade. Isso, por sua vez, tem sido uma triste realidade.

De acordo com Moreira e Baranauskas (2015) "o uso da informática aliado à constante inovação de equipamentos especialmente desenvolvidos serve como mediador no processo de ensino-aprendizagem".

A pessoa com deficiência necessita de um cuidado e educação especiais, que assegurem seu bem-estar físico, psíquico e social, ao mesmo tempo em que lhe ofereça todos os procedimentos capazes de adquirir a mesma educação e aprendizagem de uma pessoa sem deficiência.

Corrêa et al (S/D) encontram na música uma possibilidade pertinente para estabelecer a inclusão no contexto educacional autista. Para os autores, a música, entendida como um conjunto de linguagens que agem simultaneamente podem remeter a criança autista a uma realidade mais concreta, apresentando-a a um mundo ainda pouco conhecido. Na investigação, os pesquisadores constatam que a música pode instigar habilidades comportamentais das crianças, ajudando-as a desenvolver capacidades linguísticas e psíquicas.

As pessoas se sentem excluídas por não encontrarem meios que thes despertem ou lhes ajudem na prática cotidiana. Nesse sentido, a tecnologia não pode contribuir para esta exclusão, e sim ser utilizada para que o inverso aconteça. Ou seja, para que pessoas de todos os tipos de deficiência sejam incluídas no mesmo espaço social.

Nesse entorno, mostra-se pertinente a pesquisa de Zajc e Starcic (2012), que questionam justamente a tecnologia como instrumento mediador. Para tal, constatam 
que os meios tecnológicos, em sua função multimídia, é um rico recurso de inclusão, tendo em vista o leque de possibilidades que se abre a partir de seu conhecimento.

A pesquisa supracitada mostra-se convidativa no sentido de nos fazer repensar o papel inclusivo das tecnologias no contexto de inclusão social de crianças com autismo, tendo em vista que os textos tecnológicos de natureza multimídia podem ajudar a criança no processamento cerebral de informações, bem como para o controle das células cerebrais, mesmo que em escala pequena.

Diante disso, é possível afirmar que "a área da computação vem se expandindo com novas linhas de pesquisa como, por exemplo, interfaces tangíveis e música" (CORREAA et al, S/D, p. 1). Para os autores, a referida articulação serve como uma alternativa para a criação de novos mecanismos capazes de incluir pessoas com deficiência em um universo ainda repleto de limitações para as mesmas.

É necessário investir em políticas de conscientização sobre o autismo em diversos contextos sociais, mas não apenas isso. A tecnologia deve ser levada à criança autista de maneira que ela a perceba como algo prático e concreto. Para isso, é necessário desenvolver softwares específicos que possam viabilizar o uso das máquinas para pessoas com deficiência, além de incentivar a criação de ferramentas também plausíveis para que os autistas, ou outras pessoas com deficiência, possam ser efetivamente incluídos no meio em que vivem.

\section{TRABALHO FUTURO}

Durante os últimos anos, as tecnologias têm dado uma grande contribuição para a humanidade, de maneira transparente e por vezes intrusiva, nos principais ramos do mercado, inclusive na educação.

São contribuições que englobam desde as melhorias do dia a dia até a automação das grandes indústrias. Essas tecnologias proveem principalmente automatização de procedimentos já padronizados e softwares que podem auxiliar desde comércios, fábricas chegando à educação. Tornando o processo ensinoaprendizagem um pouco mais eficaz.

Mesmo com a tecnologia sendo considerada "avançada" nos dias atuais, ainda é desafiador provê educação inclusiva utilizando meios tecnológicos, ubíquos e 
pervasivos como, por exemplo, computação vestível para auxiliar na educação de pessoas com necessidades específicas, principalmente as crianças com autismo.

Para o futuro pensamos em dar ênfase no estudo da educação inclusiva, utilizando como recursos as tecnologias contemporâneas vestíveis e tangíveis, para melhorar o processo de ensino-aprendizagem de pessoas com necessidades específicas, principalmente estudar o ser humano em sua fase inicial, durante a infância e adolescência.

É importante verificar até que ponto a computação, atuando de modo transparente e integrada às atividades do cotidiano do usuário, venha a contribuir no processo ensino-aprendizagem, que engloba desde o docente até o discente. Analisar também até que ponto estas ferramentas tecnológicas podem servir apenas como auxílio, automatizando alguns métodos que outrora tenham sido utilizados de maneira clássica, sem o uso das tecnologias disponíveis e até onde elas serão fundamentais.

\section{ALGUMAS CONSIDERAÇÕES}

Diante do que foi apresentado neste artigo, é válido dizer que a tecnologia tem a capacidade de oferecer a quem a manuseia uma gama de informações que se interconectam como uma espécie de rede junto ao mundo real, o que justifica a necessidade de conhecer as ferramentas computacionais.

Misturando uma variedade considerável de linguagens, o universo da computação lida com, além da ferramenta verbal, muitos recursos imagéticos que causam sentidos e agregam significados bastante pontuais. Somado a isso, as cores também são elementos de grande valia para o desenvolvimento do conhecimento científico a respeito da computação e da tecnologia.

Assim, o conhecimento desses recursos não pode se limitar a uma microesfera social, tendo em vista a ocorrência bastante expressiva do mercado de trabalho. Logo, é necessário falar-se em medidas de inclusão tecnológica que possam agregar todos os sujeitos sociais e, com isso, compartilhar conhecimento e maximizar a inclusão, sobretudo das pessoas com qualquer tipo de deficiência, seja ela física ou intelectual.

Embora tudo na contemporaneidade pareça de fácil acesso, ainda há uma grande parcela da população brasileira que não comunga das informações 
necessárias para um manuseio satisfatório dessas ferramentas. Por vários motivos diversos, muitos brasileiros ainda não tiveram a oportunidade de se adequar a uma nova conjuntura social devido às limitações advindas da falta de acesso aos conhecimentos tecnológicos.

Por isso, o tema ora referido é de natureza fundamental no que se refere a sua contribuição ao meio acadêmico e social. Acadêmico, porque é necessário que as instituições de ensino superior sejam motivadoras de debates mais frutíferos na área, propondo medidas menos teórico-pragmáticas e mais práticas, principalmente no que tange a meios tecnológicos que já podem ser considerados básicos, como uma simples retirada de extrato bancário, a formatação elementar de um texto, o manuseio de um controle de televisão, o acesso a uma rede social dentre outros.

Em síntese, espera-se ter contribuído um pouco sobre a teoria da tecnologia como meio facilitador no processo de ensino-aprendizagem, no que se refere a uma alternativa bastante pertinente para evitar exclusão de diversas pessoas em contextos sociais bem pontuais, com foco principalmente nas pessoas com necessidades específicas.

\section{REFERÊNCIAS}

AIRES, A. C. Silva; ARAÚJO, M. V. Silva; NASCIMENTO, G. Amaral do. Autismo: convívio escolar, um desafio para a educação. 2014.

CORREAA, A. G. D. et al. O Fazer Musical de Pessoas com Deficiência: As novas tecnologias propiciando a inclusão, Laboratório de Sistemas Integráveis, São Paulo, S/D.

KLEIMAN, A. B. Modelos de Letramento e as Práticas de Alfabetização na Escola. In.: KLEIMAN, A. B. (org). Os Significados do Letramento: Uma nova perspectiva sobre a prática social de escrita. Campinas/SP: Mercado de Letras, 1995. p. 15-64.

MODALOZZO, M. O Autismo e a Convenção da ONU sobre o Direito das Pessoas com Deficiência. Revista Autismo, abril de 2011.

MOREIRA, E. A.; BARANAUSKAS, M. C. C. Tecnologias Tangíveis e Vestíveis como Recursos para Ambiente Inclusivo: Uma revisão sistemática. Anais do XXVI Simpósio Brasileiro de Informática na Educação, 2015.

OLIVEIRA, J. E. et al. Software livre como estratégia de inclusão digital: $O$ caso da UFRB. Disponível https://www.fearp.usp.br/cooperativismo/_up_imagens/(ok)_ii_ebcp_janaina_evangeli sta_de_oliveira.pdf. Acessado em 18 de jun. de 2017, às $12 \mathrm{~h} 45 \mathrm{~min}$. 
PEREIRA, B. G. Professores em Formação Inicial no Gênero Relatório de Estágio Supervisionado: Um estudo em licenciaturas paraenses. 2014. 136 f. Dissertação (Mestrado em Ensino de Língua e Literatura) - Universidade Federal do Tocantins, Araguaína, 2014.

PEREIRA, B. G. Relocalização de Saberes Acadêmicos na Construção de Vozes de Professores em Formação Inicial na Escrita Acadêmica Convencional e Reflexiva. 2016. 350 f. Tese (Doutorado em Ensino de Língua e Literatura) Universidade Federal do Tocantins, Araguaína, 2016.

SILVA, Naiane Vieira dos Reis. Letramento digital no estágio supervisionado obrigatório em ensino de língua materna. 2014. 154f. Dissertação (Mestrado em Letras: ensino de Língua e Literatura) - Universidade Federal do Tocantins, Programa de PósGraduação em Letras: ensino de Língua e Literatura, Araguaína, 2014.

SILVA, W. R. Gêneros em Práticas Escolares de Linguagens: Currículo e formação do professor. Revista Brasileira de Linguística Aplicada. 2015 (no prelo).

SILVA, W. R. Reflexão pela Escrita no Estágio Supervisionado da Licenciatura: Pesquisa em Linguística Aplicada. Campinas: Pontes Editores, 2014.

SOUZA, M. A. et al. Informática e Educação Especial: Desafios e Possibilidades Tecnológicas. Material Pedagógico da Universidade Federal Tecnológica do Paraná. Curitiba, 2008.

TEIXEIRA, A. C.; MARCON, K. Inclusão Digital: Experiências, desafios e perspectivas. Passo Fundo: Ed. Universidade de Passo Fundo, 2009.

TURCI, P. C.; COSTA, M. P. R. A Informática como Instrumento da Construção da Escola Inclusiva para Alunos com Cegueira. Revista Vozes dos Valores da UFVJM: Publicações Acadêmicas. Nº 2, V I, 2012. P. 1-24.

ZAJC, M.; STARCIC, A. I. Potentials of the Tangible User Interface (TUI) in Enhacing Inclusion of People with Special Needs in the ICT - Assisted Learning and eAcessibility. Kes-Amsta, LNAI 7327, p. 261-270, 2012.

ZANATA, E. M.; CARVALHO, D. Informática Aplicada à Educação Especial. Bauru: Universidade Estadual Paulista Júlio de Mesquita Filho, 2008. 